\title{
Smooth cutoff formulation of hierarchical reference theory for a scalar $\phi^{4}$ field theory
}

\author{
Cristian D. Ionescu, ${ }^{1, *}$ Alberto Parola, ${ }^{2}$ Davide Pini, ${ }^{1}$ and Luciano Reatto ${ }^{1}$ \\ ${ }^{1}$ Dipartimento di Fisica, Università degli Studi di Milano, Via Celoria 16, 20133 Milano, Italy \\ ${ }^{2}$ Dipartimento di Fisica e Matematica, Università dell'Insubria, Via Valleggio 11, 22100 Como, Italy
}

(Received 13 April 2007; published 12 September 2007)

\begin{abstract}
The $\phi^{4}$ scalar field theory in three dimensions, prototype for the study of phase transitions, is investigated by means of the hierarchical reference theory (HRT) in its smooth cutoff formulation. The critical behavior is described by scaling laws and critical exponents which compare favorably with the known values of the Ising universality class. The inverse susceptibility vanishes identically inside the coexistence curve, providing a first principle implementation of the Maxwell construction, and shows the expected discontinuity across the phase boundary, at variance with the usual sharp cutoff implementation of HRT. The correct description of first and second order phase transitions within a microscopic, nonperturbative approach is thus achieved in the smooth cutoff HRT.
\end{abstract}

DOI: 10.1103/PhysRevE.76.031113 PACS number(s): 64.60.Fr, 64.60.Cn, 64.60.Ak, 05.70.Fh

\section{INTRODUCTION}

The equilibrium properties of classical fluids have been extensively studied in the past decades and very successful theories of both the gas and the liquid states are available by now $[1,2]$. However, a fully satisfactory description of the liquid-vapor phase transition within a microscopic liquid state approach has still to come. Although the qualitative features and the universal properties characterizing the transition are known since the development of the renormalization group (RG) approach [3], a quantitative theory able to predict phase boundaries for specific fluid models is not avaliable yet: Most of the theories developed in liquid-state physics deal mainly with short-range correlations and shortwavelength density fluctuations. That is why they are not able to reproduce, even qualitatively, the phenomenology of the critical region. Furthermore, these liquid-state theories give an unsatisfactory description of the thermodynamics along the first order liquid-vapor transition line. The convexity of the free energy is not guaranteed in the integral equation approach or in mean-field theories and in fact is always violated inside the two-phase region. This intrinsic deficiency is usually overcome by means of ad hoc procedures, like Maxwell double tangent construction. An accurate description of the long-wavelength fluctuations is implemented within the liquid-state framework in the hierarchical reference theory of fluids (HRT) [4,5], where the basic RG concept of selective turning on of fluctuations is built into a genuine liquid-state theory. This feature made HRT the only practical scheme able to determine both universal and nonuniversal critical properties of fluids, so far. Moreover, the proper treatment of long-wavelength fluctuations forces the free energy to display the correct convexity also in the twophase region, thereby incorporating Maxwell construction: A major achievement of HRT. Although quite successful, the current sharp cutoff formulation of HRT predicts a diverging compressibility along the binodal (in any dimension smaller

\footnotetext{
*On leave of absence from the INFPLR-ISS, P.O. Box MG-23, RO 76911 Bucharest, Romania.
}

than four) [6] while, for a scalar order parameter, a finite value of the inverse compressibility at coexistence is expected. In this paper, we show that this failure of the HRT approach in the sharp cutoff formulation can be overcome by studying the smooth cutoff implementation, already introduced several years ago [7]. In order to clarify the qualitative properties expected within this approach, we consider the simplest model displaying a phase transition within the Ising universality class: The scalar $\phi^{4}$ field theory in three dimensions. A somehow related formalism has been recently put forward by Caillol [8] who developed a nonperturbative renormalization-group theory for fluids, within the grand canonical ensemble, and applied it to the Kac model.

The paper is organized as follows: In Sec. II we review the sharp and the smooth cutoff formalism for a general microscopic fluid model. In Sec. III this formalism is applied to a $\phi^{4}$ field theory, where several simplifications occur. An approximate closure relation is introduced and discussed. The numerical solution of the equations at low temperatures and the comparison between the two approaches is presented in Sec. IV, where the behavior at coexistence is analyzed. The critical properties of HRT in the smooth cutoff formulation are derived in Sec. V, while Sec. VI contains a short summary of the results and some perspective.

\section{HRT EQUATIONS IN SHARP AND SMOOTH CUTOFF FORMULATIONS}

The common principle underlying HRT, in its various formulations, is the selective treatment of density fluctuations on different length scales. Starting from a mean field approximation, the effects of density fluctuations on short wavelengths are included first. This is accomplished by turning on the attractive part of the potential progressively, starting from its Fourier components with larger wave vectors. A key role is therefore played by the cutoff wave vector $Q$ which separates the Fourier components already taken into account (those with $k>Q$ ) from those ignored $(k<Q)$. The full spectrum of fluctuations is then included only in the $Q$ $\rightarrow 0$ limit, while at $Q \rightarrow \infty$ the mean field approximation is recovered. The variation in the free energy induced by an 
infinitesimal change in $Q$ can be computed exactly by use of perturbation theory, leading to a differential "evolution" equation for the thermodynamics of the system.

We study a system of particles in dimension $d$, interacting via a two-body potential $v(r)$ formally written as the sum of two contributions:

$$
v(r)=v_{R}(r)+w(r)
$$

where $v_{R}(r)$ is a short-range, repulsive reference part and $w(r)$ is a mostly attractive term defined by a regular function which can be Fourier transformed. The properties of the system interacting via $v_{R}$ are assumed to be known by other liquid state theories or simulations. In order to implement the principle of HRT, the Fourier components of $w(r)$ have to be included gradually. Therefore, we define a sequence of $Q$ systems characterized by an interaction $v_{Q}(r)$ :

$$
v_{Q}(r)=v_{R}(r)+w_{Q}(r),
$$

interpolating between the reference system [obtained for $w_{Q}(r)=0$ ] and the fully interacting model [ for $\left.w_{Q}(r)=w(r)\right]$. The difference between sharp and smooth cutoff formulation resides in the way the cutoff on the Fourier components of the potential is implemented, as discussed in the following.

\section{A. Sharp cutoff}

In the sharp cutoff formulation, the precise definition of $w_{Q}$ is conveniently given in Fourier space:

$$
\widetilde{w}_{Q}(k)=\left\{\begin{array}{cl}
\widetilde{w}(k) & \text { for } k>Q, \\
0 & \text { for } k<Q,
\end{array}\right.
$$

where the tilde denotes Fourier transform. Here, the cutoff $Q$ varies between $Q=\infty$, where the potential reduces to the reference part, and $Q=0$, where the physical interaction (1) is recovered. Due to the long range oscillating tail in $w_{Q}(r)$, induced by the sharp cutoff, no phase transition occurs in each $Q$ system until the $Q \rightarrow 0$ limit is attained. It is now possible to write a differential equation for the evolution of the free energy $A_{Q}$ of the $Q$ system when the cutoff is varied. This equation, though exact, is not closed because it involves the two body correlation function of the $Q$ system, and suitable approximations linking thermodynamics and correlations must be introduced. Before discussing the adopted closure, following the RG approach, it is convenient to introduce an additional definition in order to take into account the Fourier component of the potential with vanishing wave vector since the very beginning of the integration procedure. The $k=0$ term in fact gives rise to the mean-field contribution to the free energy which provides a physically correct starting point for the description of phase transitions. This is achieved by introducing a modified free energy density defined as

$$
\begin{aligned}
\mathcal{A}_{Q}= & -\frac{\beta}{V} A_{Q}-\frac{1}{2} \rho\left[\phi(r=0)-\phi_{Q}(r=0)\right]+\frac{\rho^{2}}{2}[\widetilde{\phi}(k=0) \\
& \left.-\widetilde{\phi}_{Q}(k=0)\right],
\end{aligned}
$$

where $\phi(r)=-\beta w(r), \beta=1 / k_{B} T$, and $\rho$ is the particle density.
Analogously, the modified direct correlation function includes the random phase approximation (RPA) contribution:

$$
\mathcal{C}_{Q}(k) \equiv \widetilde{c}_{Q}(k)+\widetilde{\phi}(k)-\widetilde{\phi}_{Q}(k),
$$

where $c_{Q}(r)$ is the usual direct correlation function [1] of the $Q$ system (with the inclusion of the ideal gas term). The evolution of the (modified) free energy satisfies the exact differential equation:

$$
\frac{d \mathcal{A}}{d Q}=-\frac{d}{2} \Omega_{d} Q^{d-1} \ln \left(1-\frac{\widetilde{\phi}(Q)}{\mathcal{C}_{Q}(Q)}\right),
$$

where $\Omega_{d}$ is the volume of the unit sphere in $d$ dimensions divided by $(2 \pi)^{d}$. An analogous equation governs the evolution of $\mathcal{C}_{Q}(k)$ which, however, depends on the three and four body correlation functions of the $Q$ system, thereby giving rise to an exact hierarchy of differential equations. In the following section we will provide an approximate closure at the level of the first equation of the hierarchy, Eq. (6).

\section{B. Smooth cutoff}

In the smooth cutoff formulation of HRT [7] the discontinuity in the Fourier transform of $w_{Q}$ induced by the definition (3) is removed by the alternative choice

$$
\widetilde{w}_{t}(k)=\widetilde{w}(k)-e^{-2 t} \widetilde{w}\left(k e^{t}\right),
$$

which identically vanishes at $t=0$ and tends to $\widetilde{w}(k)$ for $t$ $\rightarrow \infty$. If $\widetilde{w}(k)$ varies on the characteristic wave vector scale $\Lambda$, the quantity $Q=\Lambda e^{-t}$ acts as an effective cutoff wave vector: The Fourier components of $\widetilde{w}_{t}(k)$ for $k<Q$ are efficiently suppressed and the long range repulsive tail present in $w_{t}(r)$ contrasts the tendency towards phase separation at any finite $t$. The sequence of intermediate potentials $\widetilde{w}_{t}(k)$ defined in Eq. (7) belongs to the class studied in Ref. [8] and corresponds to the particular choice of cutoff function $R_{t}(k) \widetilde{w}(k)=e^{-2 t} \widetilde{w}\left(k e^{t}\right)\left[\widetilde{w}(k)-e^{-2 t} \widetilde{w}\left(k e^{t}\right)\right]^{-1}$. The requirements stated in [8] are satisfied by $R_{t}(k)$ provided $\widetilde{w}(k)$ decays rapidly at large wave vectors. First order perturbation theory provides the change in the free energy induced by an infinitesimal increase of the parameter $t$. By use of the same definition (4) already introduced, the exact evolution equation of the (modified) free energy in the smooth cutoff formulation of HRT reads

$$
\frac{d \mathcal{A}_{t}}{d t}=\frac{1}{2} \int \frac{d^{d} k}{(2 \pi)^{d}} F_{t}(k) \frac{d \widetilde{\phi}_{t}(k)}{d t}
$$

where $\widetilde{\phi}_{t}(k)=-\beta \widetilde{w}_{t}(k)$ and the two point function $F_{t}(k)=$ $-\left[\widetilde{c}_{t}(k)\right]^{-1}$ is just $\rho$ times the structure factor $S_{Q}(k)$ of the $Q$ system. As usual, it is more convenient to introduce the modified direct correlation function $\mathcal{C}_{t}$ [Eq. (5)] which includes the total interparticle potential in mean-field approximation, because we expect that its convergence properties as $t \rightarrow \infty$ will be better than those of the "bare" correlations $\widetilde{c}_{t}(k)$. By use of Eqs. (5) and (7) we get a formal expression for the bare two point function appearing in Eq. (8): 


$$
F_{t}(k)=-\left[\mathcal{C}_{t}(k)-\widetilde{\phi}(k)+\widetilde{\phi}_{t}(k)\right]^{-1}=-\left[\mathcal{C}_{t}(k)-e^{-2 t} \widetilde{\phi}\left(k e^{t}\right)\right]^{-1} .
$$

Analogous to the sharp cutoff case, the flow equation (8) for $\mathcal{A}_{t}$ is not closed and a suitable approximation for $\mathcal{C}_{t}(k)$ must be introduced.

\section{HRT EQUATIONS FOR THE $\phi^{4}$ SCALAR FIELD THEORY}

In order to test the accuracy of HRT in the description of phase transitions, we specialize the general equations (6) and (8) to a particular model of fluid. We first note that, in systems with regular short-range attractive interactions, the Fourier transform of $\phi(r)$ is a positive, monotonic decreasing function of the wave vector, quadratic in $k$ at small $k$. The simplest model with these features is defined by an effective interaction of the form

$$
\widetilde{\phi}(k)=\left\{\begin{array}{cc}
b\left(\Lambda^{2}-k^{2}\right) & \text { for } k<\Lambda, \\
0 & \text { for } k>\Lambda .
\end{array}\right.
$$

By substituting this form into Eqs. (6) and (8) we get, respectively,

$$
\begin{gathered}
\frac{d \mathcal{A}_{Q}}{d Q}=-\frac{d}{2} \Omega_{d} Q^{d-1} \ln \left(1-\frac{b\left(\Lambda^{2}-Q^{2}\right)}{\mathcal{C}_{Q}(Q)}\right), \\
\frac{d \mathcal{A}_{Q}}{d Q}=b Q \int_{k<Q} \frac{d^{d} k}{(2 \pi)^{d}} \frac{1}{\mathcal{C}_{Q}(k)+b\left(k^{2}-Q^{2}\right)},
\end{gathered}
$$

where the cutoff wave vector in the smooth cutoff equation (12) is defined as $Q \equiv \Lambda e^{-t}$. The equations hold for $Q<\Lambda$ and must be supplemented by the initial condition at $Q=\Lambda$, where $\mathcal{A}_{Q}$ reduces to the mean field expression. In both cases, as initial condition, we take a Landau-Ginzburg quartic form, centered around the critical density $\rho_{c}$, with coupling constants $(r, u)[3]$ :

$$
\mathcal{A}_{\Lambda}(\rho)=\mathcal{A}_{\Lambda}\left(\rho_{c}\right)-r\left(\rho-\rho_{c}\right)^{2}-u\left(\rho-\rho_{c}\right)^{4} .
$$

In a fully microscopic model, defined by a realistic two body interaction $w(r)$, we expect that the quartic form (13) represents the physical free energy density in a small neighborhood of the critical density $\rho_{c}$. In this case, the effective couplings $(r, u)$ are functions of the temperature and may be estimated in mean field theory. Short range density fluctuations, corresponding to wave vectors $Q>\Lambda$, are expected to renormalize the bare values, as shown in Ref. [9] where this problem is examined within the HRT formalism. The modified direct correlation function appearing in the two evolution equations (11) and (12) is approximated in the spirit of the local potential approximation of $\mathrm{RG}[3,4]$, by an Ornstein-Zernike form

$$
\mathcal{C}_{Q}(k)=\mathcal{C}_{Q}(0)-b k^{2}=\frac{\partial^{2} \mathcal{A}_{Q}}{\partial \rho^{2}}-b k^{2} .
$$

In Eq. (14) the range of the direct correlation function has been assumed to coincide with that of the chosen potential
(10) with no renormalization due to fluctuations. This is actually the only approximation introduced into the HRT formalism and implies an analytic momentum dependence of the correlation functions in the whole phase diagram, critical point included. In order to allow for a nonvanishing critical exponent $\eta$ within HRT, we must go beyond the parametrization (14) taking into account the effects of fluctuations on the range of the correlation function. This can be accomplished by examining the second equation of the HRT hierarchy, governing the evolution of $\mathcal{C}_{Q}(k)$ [4]. In fact, it has been shown [7] that, in the critical region and in the limit $Q \rightarrow 0$, this equation allows one to reproduce correctly the critical exponents up to the $\epsilon^{2}$ term in the $\epsilon=4-d$ expansion, hence giving a nonvanishing value of $\eta$. The last equality in Eq. (14) directly follows from the compressibility sum rule [1], according to which the structure factor evaluated at zero wave vector is equal to the reduced compressibility of the system. By use of this parametrization, Eqs. (11) and (12) give rise to a couple of partial differential equations describing the effect of fluctuations into the mean field free energy within the sharp and smooth cutoff formulation of HRT for a potential of the form (10). The resulting HRT equations are then

$$
\begin{gathered}
\frac{d \mathcal{A}_{Q}}{d Q}=-\frac{d}{2} \Omega_{d} Q^{d-1} \ln \left[\frac{-\mathcal{A}_{Q}^{\prime \prime}+b \Lambda^{2}}{-\mathcal{A}_{Q}^{\prime \prime}+b Q^{2}}\right], \\
\frac{d \mathcal{A}_{Q}}{d Q}=-\frac{b \Omega_{d} Q^{d+1}}{-\mathcal{A}_{Q}^{\prime \prime}+b Q^{2}}
\end{gathered}
$$

for the sharp and smooth cutoff, respectively, where primes mean differentiation with respect to $\rho$. The sharp cutoff equation (15) for a $\phi^{4}$ field theory has already been studied in Refs. [5,6] both in the critical region and at phase coexistence where, for $Q \rightarrow 0$, the term $-\mathcal{A}_{Q}^{\prime \prime}+b \Lambda^{2}$ in the argument of the logarithm can be neglected. We remark that this does not affect by any means the universal behavior at criticality or the qualitative features of the first-order transition discussed here. In Ref. [10] the smooth cutoff RG equations have been derived and studied for a $\phi^{4}$ field theory. The coincidence, after a trivial rescaling, of our HRT equation (16) with Eq. (2.6) of Ref. [10] for the special cutoff choice $n=d / 2+1$ shows that indeed (i) the parabolic potential model defined by Eq. (10) is equivalent to a $\phi^{4}$ scalar field theory and (ii) that the smooth cutoff HRT formalism becomes equivalent to the RG in the scaling limit [11].

\section{COEXISTENCE BOUNDARY}

A fully implicit predictor-corrector finite difference algorithm is used in order to solve the HRT evolution equations (15) and (16) for a $\phi^{4}$ field theory. The initial condition (13) is imposed at $Q=\Lambda$, consistently with the assumed form of the interaction potential (10). The inverse compressibility $\chi^{-1}=-\partial^{2} \mathcal{A}_{Q} / \partial \rho^{2}$ at the end of integration (i.e., $Q \rightarrow 0$ ) is shown in Fig. 1 as a function of $\rho-\rho_{c}$ for a representative choice of parameters in the broken-symmetry regime. At large densities the two formulations of HRT provide very similar results. Moreover, both show a region of infinite 


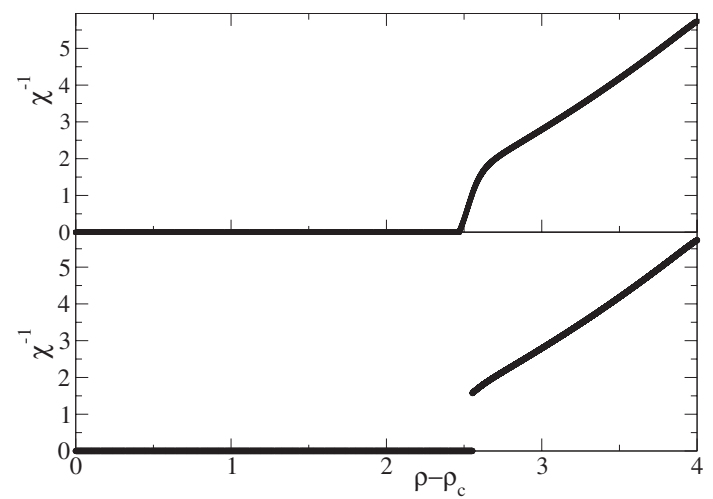

FIG. 1. Inverse compressibility as a function of the order parameter $\rho-\rho_{c}$ in three dimensions from the sharp (above) and smooth cutoff formulation (below). Units are set by $b \Lambda^{2}=1$ and $\Omega_{3} \Lambda^{3}=1$, while the coupling constants are $r=-0.47$ and $u=0.0035$. Upper panel: Result from the sharp cutoff formulation, Eq. (15). Lower panel: Result from the smooth cutoff formulation, Eq. (16). Notice the presence of the expected discontinuity across the coexistence boundary in the smooth cutoff case.

compressibility, consistent with the convexity requirement of the free energy. The important difference between the smooth and the sharp cutoff formulation is the presence of a discontinuity across the coexistence curve in three dimensions in the smooth cutoff case, in agreement with the expected behavior for a scalar order parameter. Instead, as already discussed in Ref. [6], the sharp cutoff HRT equation predicts the divergence of the compressibility when coexistence is approached.

We now provide an analytical interpretation of the origin of the discontinuity following the procedure devised in [6], for the sharp cutoff formalism. We first write our Eq. (16) in a scale invariant form. By applying the rescaling

$$
\begin{gathered}
x=\sqrt{\frac{b}{\Omega_{d}} \rho,} \\
f=-\frac{\mathcal{A}_{Q}}{\Omega_{d}}
\end{gathered}
$$

to Eq. (16), we obtain

$$
Q \frac{\partial f}{\partial Q}=\frac{Q^{d+2}}{f_{x x}+Q^{2}},
$$

where we have set $f_{x x}=\partial^{2} f / \partial x^{2}$. By performing the substitution

$$
\phi=\frac{1}{f_{x x}+Q^{2}}
$$

and taking the double derivative of Eq. (18) with respect to $x$, the equation is written in quasilinear form, suitable for an analytical study:

$$
\frac{1}{\phi^{2}} \frac{\partial \phi}{\partial Q}+2 Q=-Q^{d+1} \phi_{x x}
$$

In the two-phase region, the observed convexity of the free energy implies the divergence of the compressibility $\chi$. This means that for $Q \rightarrow 0, f_{x x} \propto \chi^{-1}$ vanishes and $\phi \rightarrow+\infty$. In this case, we can neglect the first term of Eq. (20), so that

$$
\phi_{x x}=-2 Q^{-d} \text {, }
$$

whose solution is

$$
\phi=\left(x_{0}^{2}-x^{2}\right) Q^{-d},
$$

where the integration constant $x_{0}$ plays the role of rescaled coexistence density. Indeed, this expression shows that $\phi$ $\rightarrow+\infty$ as $Q \rightarrow 0$, consistently with our assumption, only for $|x|<x_{0}$ : HRT correctly predicts the existence of a finite region of infinite compressibility, but our analysis is not able to describe the behavior of $\phi$ across the phase boundary, i.e., the transition between a finite solution for $|x|>x_{0}$ and the asymptotic form (22) inside the binodal. Following [6], it is useful to zoom-in the region close to $x_{0}$ by rescaling the $x$ variable as

$$
z=\left(x-x_{0}+a Q^{2}\right) Q^{-d} .
$$

The additive term $a Q^{2}$ takes into account the fluctuation corrections to the position of the phase boundary, which are known to play an important role in determining the asymptotic solution on the binodal [6]. Then Eq. (20) becomes

$$
\frac{1}{\phi^{2}}\left[Q \frac{\partial \phi}{\partial Q}-\left(d z-2 a Q^{2-d} \frac{\partial \phi}{\partial z}\right)\right]+2 Q^{2}=-Q^{2-d} \frac{\partial^{2} \phi}{\partial z^{2}} .
$$

By keeping only the dominant terms as $Q \rightarrow 0$ in Eq. (24), we obtain the following fixed-point equation:

$$
\frac{2 a}{\phi^{2}} \frac{\partial \phi}{\partial z}=-\frac{\partial^{2} \phi}{\partial z^{2}} \text {. }
$$

Once integrated, the equation gives

$$
\phi+\frac{2 a}{c} \ln (\phi c-2 a)=-c z+k
$$

where $c, k$ are integration constants. Inside the coexistence region, i.e., for $z \rightarrow-\infty$, Eq. (26) must match Eq. (22) which, expressed in terms of $z$, reads

$$
\phi=-\left(x+x_{0}\right) z \approx-2 x_{0} z .
$$

This sets the constant $c$ at the value $c=2 x_{0}$. When the coexistence boundary is approached from outside, i.e., for $z \rightarrow$ $+\infty$, Eq. (26) predicts a finite compressibility: $\phi \rightarrow 2 a / c$ $\equiv \phi_{0}$. We can then rewrite Eq. (26) as

$$
\phi+\phi_{0} \ln \left(\phi / \phi_{0}-1\right)=-2 x_{0} z+\text { const },
$$

which describes the asymptotic behavior of the solution across the phase boundary. A numerical verification of such a scaling law can be obtained by considering the derivative of Eq. (28) with respect to $z$ : 


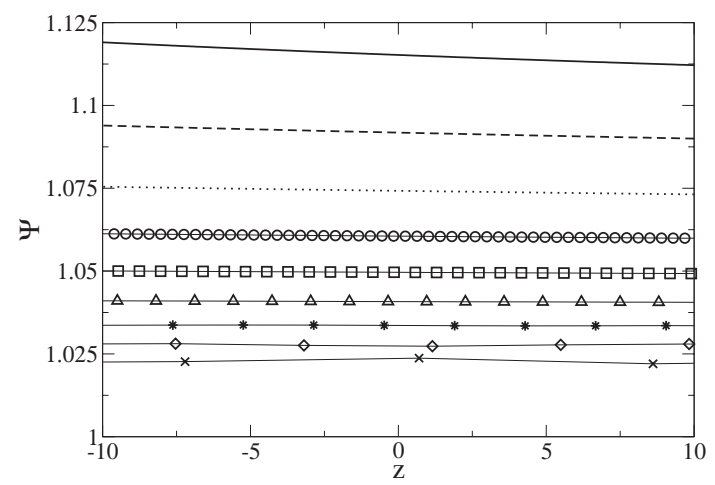

FIG. 2. Numerical results for the quantity $\Psi$ of Eq. (29). Different lines correspond to different $Q$ values. From top to bottom: $\ln Q=-4.0$ (solid line), $\ln Q=-4.2$ (dashed line), $\ln Q=-4.4$ (dotted line), $\ln Q=-4.6$ (circles), $\ln Q=-4.8$ (squares), $\ln Q=-5.0$ (triangles), $\ln Q=-5.2$ (stars), $\ln Q=-5.4$ (diamonds), and $\ln Q=-5.6$ (crosses).

$$
\Psi=\frac{\partial \phi}{\partial z} \frac{1}{2 x_{0}} \frac{\phi}{\phi-\phi_{0}}=1 .
$$

The validity of this equation has been tested on the numerical solution of Eq. (16) by selecting small $Q$ values $(Q<2$ $\times 10^{-2}$ ) and mesh points extremely close to the phase boundary, so that the rescaled variable $z$ given in Eq. (23) is $O(1)$. The results are shown in Fig. 2, where several sets of data points, corresponding to different $Q$ 's and different $x$ 's, are shown. The asymptotic collapse of the data towards unity, as required by Eq. (29), can be appreciated. The results shown in the figure were obtained with a mesh $\Delta \rho=4 \times 10^{-7}$. Probing the asymptotic regime further would have required an even smaller $\Delta \rho$, so as to sample the region $z=O(1)$ for $Q$ smaller than those considered in the figure. The discontinuity of $\chi^{-1}$ across the binodal, shown in Fig. 1, is therefore a genuine consequence of the smooth cutoff HRT formalism. The analysis has been performed for a particular choice of the potential (10) and for a closure of the form (14), because these assumptions considerably simplify the evolution equation (18). We also studied the smooth cutoff HRT equation for a realistic Yukawa interaction $w(r)$, allowing for a difference between the range of the potential and that of the direct correlation function. The numerical solution in the asymptotic $Q \rightarrow 0$ regime shows the expected jump in the inverse susceptibility across the coexistence boundary. Our findings are consistent with the numerical analysis of Ref. [10], where the behavior of $\chi^{-1}$ has been investigated for a one-parameter family of smooth cutoff RG theories which includes, as a limiting case, also the sharp cutoff form (15), besides our Eq. (16): The discontinuity is present for all values of the parameter and vanishes only in the sharp cutoff limit.

\section{CRITICAL REGION}

In Sec. III we mentioned that the smooth cutoff HRT equation for a parabolic potential (10) reduces to a known
RG structure, analogously to the sharp cutoff case [4]. It is therefore interesting to evaluate the critical exponents, by studying the fixed point solution of the HRT equation and compare with the direct numerical solution of Eq. (16). Following the usual HRT procedure [5,7], inspired by the RG approach, we first rescale the variables $\mathcal{A}_{t}(\rho)$ and $\rho$ (at fixed temperature $T$ ), in order to blow up the critical region:

$$
\begin{gathered}
H(y, Q)=\frac{1}{\Omega_{d}} Q^{-d}\left[\mathcal{A}_{Q}\left(\rho_{c}\right)-\mathcal{A}_{Q}(\rho)\right], \\
y=\sqrt{\frac{b}{\Omega_{d}}} Q^{(2-d) / 2}\left(\rho-\rho_{c}\right),
\end{gathered}
$$

where $\rho_{c}$ is the critical density. By performing the substitution we get

$$
-Q \frac{\partial H}{\partial Q}+\frac{d-2}{2} y H^{\prime}-d H=\frac{1}{H_{0}^{\prime \prime}+1}-\frac{1}{H^{\prime \prime}+1},
$$

where $H_{0}^{\prime \prime}$ is the the second derivative of $H(y)$ evaluated at the critical density. We first look for a fixed point solution of Eq. (31), i.e., a solution $H^{*}(y)$ independent of $Q$. According to our definition, $H(0, Q)=0$ and then also $H^{*}(0)=0$. The fixed point solution is an even function of $y$, so that $H^{*{ }^{\prime \prime}}(0)$ $=0$ while $H^{* \prime \prime}$ [which explicitly appears in Eq. (31)] has to be tuned in such a way that the fixed point solution $H^{*}(y)$ is regular on the whole real axis. Numerically, it is more convenient to write the equation for the quantity $\mu(y)=H^{* \prime}(y)$, which can be obtained by differentiation of Eq. (31) with respect to $y$ :

$$
\frac{d-2}{2} y \mu^{\prime}-\frac{d+2}{2} \mu=\frac{\mu^{\prime \prime}}{\left(\mu^{\prime}+1\right)^{2}},
$$

with initial conditions $\mu(0)=0$ and $\mu^{\prime}(0)=p$. Equation (32) admits a single regular solution for a unique choice of $p$ [besides the trivial Gaussian solution $\mu(y)=0$ which corresponds to $p=0]$. By numerical integration of the fixed point equation (32) using a trial and error method we found a regular solution for $p=-0.186 \ldots$ By setting $H(y, Q)$ $=H^{*}(y)+h(y) Q^{-\lambda}$ and linearizing the evolution equation (31) for small $h$, we obtain the eigenvalue equation:

$$
\frac{d-2}{2} y h^{\prime}+(\lambda-d) h=-\frac{h_{0}^{\prime \prime}}{\left(\mu_{0}^{\prime}+1\right)^{2}}+\frac{h^{\prime \prime}}{\left(\mu^{\prime}+1\right)^{2}},
$$

where again $h_{0}^{\prime \prime}$ is the second derivative of the eigenfunction evaluated at $y=0$. By shifting the eigenfunction $h(y) \rightarrow h(y)$ + const, the constant term in Eq. (33) can be eliminated. The eigenvalue $\lambda$ is determined by the requirement that $h(y)$ is free from singularities at all $y$ 's. Relevant perturbations, driving the system out of the critical point, correspond to positive eigenvalues. All the eigenfunctions can be classified according to their parity with respect to the symmetry $y \rightarrow-y$ : Even solutions describe perturbations along the critical isochores, i.e., governing the temperature dependence of the free energy in the critical region, while odd eigenfunctions correspond to changes along the density axis. As usual, a single relevant odd eigenfunction is present: $h(y)=H^{* \prime}(y)$ with associated 


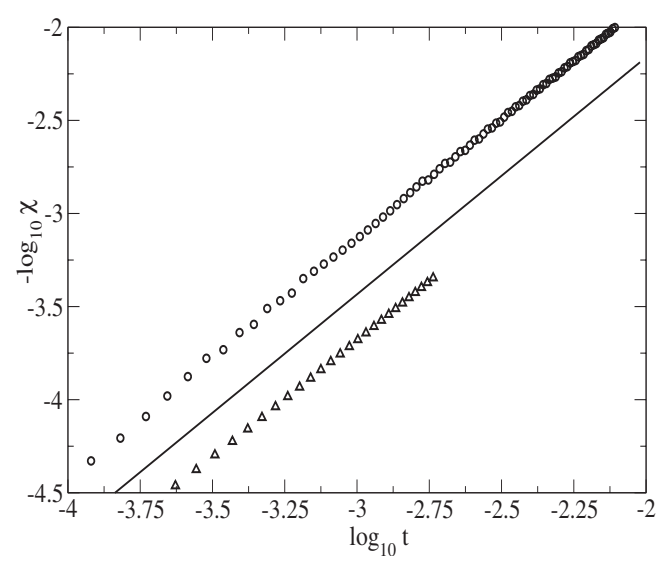

FIG. 3. A log-log plot of the inverse dimensionless compressibility as obtained by numerical integration of Eq. (16) as a function of the reduced "temperature" $t=\left|r-r_{c}\right|$. Triangles refer to the critical isochore, above the critical temperature $r>r_{c}$. Circles correspond to data along the coexistence curve for $r<r_{c}$. A linear fit gives an exponent $\gamma=1.28 \pm 0.02$ close to the result obtained from the fixed point analysis $\gamma=1.300 \pm 0.001$ (solid line).

eigenvalue $\lambda=(d-2) / 2$ [4] which implies, via scaling relations, a critical exponent $\eta=0$, in agreement with the assumed analyticity of the correlation function (14). The numerical solution of the eigenvalue equation (33) shows that a single relevant even eigenfunction is present, corresponding to an eigenvalue $\lambda=1.539 \pm 0.001$ in three dimensions. The exponent $\gamma$ governing the divergence of the compressibility $\chi \sim\left|T-T_{c}\right|^{-\gamma}$ is then given by $\gamma=2 / \lambda=1.300 \pm 0.001$, to be compared with the accepted value for the Ising universality class $\gamma \sim 1.24$. This result agrees, within numerical uncertainties, with the estimate of $\gamma$ extracted from the numerical solution of the smooth cutoff HRT equation (16), as shown in Fig. 3. Since the usual scaling relations among critical exponents are satisfied by HRT and the approximate OrnsteinZernike closure (14) implies that the scaling exponent $\eta$ is vanishing, knowledge of $\gamma$ is sufficient to determine all the remaining critical exponents. In particular, the critical exponent $\beta$ describing the shape of the coexistence curve $(\mid \rho$ $\left.-\rho_{c}|\sim| T-\left.T_{c}\right|^{\beta}\right)$ is obtained by use of the scaling relations [4], which give $\beta=\gamma / 4 \simeq 0.325$. This value is consistent with the fit of the numerical results, as shown in Fig. 4. Contrary to the prediction of HRT in the sharp cutoff formulation, the smooth cutoff equations lead to a divergence of the specific heat at criticality: $C_{V} \sim\left|T-T_{c}\right|^{-\alpha}$. The critical exponent can

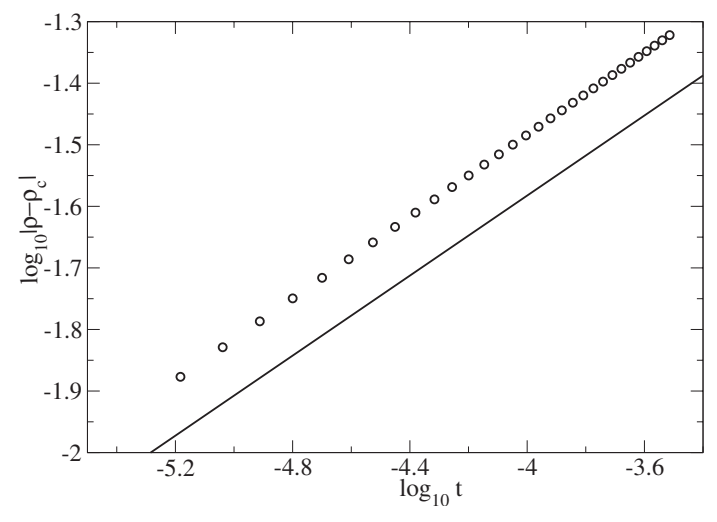

FIG. 4. Location of the coexistence curve as obtained by numerical integration of Eq. (16) as a function of the reduced "temperature" $t=\left|r-r_{c}\right|$. A linear fit gives $\beta=0.33 \pm 0.03$, consistent with the result from the fixed point analysis $\beta \simeq 0.325$ (solid line).

be evaluated by use of scaling laws with the result $\alpha=(4$ $-3 \gamma) / 2 \approx 0.050$. In Table I the values of the critical exponents in a three-dimensional fluid according to different approximations are reported. "Exact" values are derived from extrapolation of high-temperature series expansions [12], while the sharp cutoff HRT results have been obtained in [5]. In both HRT formulations the exponent $\eta$ vanishes because of the $\mathrm{OZ}$ assumption, and $\delta=5$ follows from scaling.

\section{CONCLUSIONS}

The smooth cutoff HRT equation (8) has been derived in the framework of liquid state theory for a general fluid model. The theory has been then specialized to a "parabolic potential" which corresponds to a microscopic realization of a $\phi^{4}$ theory. The resulting partial differential equation turns out to be equivalent to a particular formulation of smooth cutoff RG theory [10]. We stress that, contrary to other RG methods, the gradual turning on of fluctuations which characterizes the HRT approach, is performed directly on the physical quantities, like the free energy and no a priori mapping to effective models is required. This allows to preserve the information on both the universal and the nonuniversal properties of the system. The equation has been numerically solved both above and below the critical temperature showing that smooth cutoff HRT provides an extremely promising tool for the description of the phase diagram of fluids.

TABLE I. HRT estimates of the critical exponents for the Ising universality class in three dimensions compared to the exact values [12] obtained by extrapolation of high-temperature series expansions. Sharp cutoff HRT results from Ref. [4].

\begin{tabular}{lccc}
\hline \hline Exponent & $\begin{array}{c}\text { "Exact" } \\
\text { value }\end{array}$ & $\begin{array}{c}\text { Sharp } \\
\text { cutoff HRT }\end{array}$ & $\begin{array}{c}\text { Smooth } \\
\text { cutoff HRT }\end{array}$ \\
\hline$\alpha$ & 0.110 & -0.07 & 0.05 \\
$\beta$ & 0.327 & 0.345 & 0.330 \\
$\gamma$ & 1.237 & 1.378 & 1.300 \\
$\delta$ & 4.789 & 5 & 5 \\
$\eta$ & 0.036 & 0 & 0 \\
\hline \hline
\end{tabular}


Among the unique features of this formalism we recall the following.

A treatment of the critical region consistent with scaling laws and characterized by nonclassical critical exponents in good agreement with the exact values.

A built-in mechanism leading to the convexity of the free energy which implements Maxwell's construction via the inclusion of long range density fluctuations.

An accurate description of the first order transition, which predicts the correct jump of the inverse compressibility at the phase boundary.

Although in this paper we used the smooth cutoff HRT for the analysis of a coarse-grained Hamiltonian, the $\phi^{4}$ field theory, Eq. (8) can be directly applied to fully microscopic models of fluids, as already shown in the sharp cutoff case (see [4] and references therein). A particularly favorable choice may be the Yukawa fluid, which allows for an exact implementation of the core condition for the whole sequence of intermediate models (7) interpolating between the reference and the fully interacting systems. This feature can make the smooth cutoff formulation a valuable tool for the theoretical investigation of the phase behavior of simple and complex fluids.

\section{ACKNOWLEDGMENT}

We acknowledge support from the Marie Curie program of the European Commission, Contract No. MRTN-CT2003504712 .
[1] J. P. Hansen and I. R. McDonald, Theory of Simple Liquids, 3rd ed., (Academic Press, London 2006).

[2] C. Caccamo, Phys. Rep. 274, 1 (1996).

[3] K. G. Wilson and J. B. Kogut, Phys. Rep., Phys. Lett. 12, 75 (1974).

[4] A. Parola and L. Reatto, Adv. Phys. 44, 211 (1995).

[5] A. Parola and L. Reatto, Phys. Rev. Lett. 53, 2417 (1984); Phys. Rev. A 31, 3309 (1985).

[6] A. Parola, D. Pini, and L. Reatto, Phys. Rev. E 48, 3321 (1993).
[7] A. Parola, J. Phys. C 19, 5071 (1986).

[8] J. M. Caillol, Mol. Phys. 104, 1931 (2006).

[9] A. Brognara, A. Parola, and L. Reatto, Phys. Rev. E 64, 026122 (2001).

[10] A. Bonanno and G. Lacagnina, Nucl. Phys. B 693, 36 (2004).

[11] The equivalence between the asymptotic sharp cutoff HRT equations and an implementation of RG for the $\phi^{4}$ theory has been already proved in Refs. [5].

[12] A. Pelissetto and E. Vicari, Phys. Rep. 368, 549 (2002). 